\title{
La Littérature Comparée: Une Voix Qui Se Soulève Dans Le Contexte De La Globalisation
}

\author{
Maria Regina Barcelos Bettiol
}

\begin{abstract}
RESUMO: A Literatura Comparada ao confrontar diferentes literaturas, disciplinas, em colocar em perspetiva o local e o nacional, tem nos mostrado que a literatura, ainda que em contexto de globalização/mundialização, continua a ser um espaço de resistência, ou seja, o texto literário não é totalmente subserviente aos modelos impostos pela globalização/mundialização, o texto literário tem sempre um olhar crítico e deformador em relação a esses modelos.
\end{abstract}

Palavras-chave: literatura comparada; literatura e mundo; Welt-humanismo; globalização.

Literatura nacional não quer hoje dizer coisa muito importante: chegamos ao momento da literatura mundial e todos devemos contribuir para apressar o advento de tal época.

Johann Wolfgang Von Goethe (Conversações de Goethe com Eckermann)

\begin{abstract}
La globalisation provoque un trouble profond dans notre sentiment d'appartenance, et dans notre confiance en l'avenir. Certes, ce cadre globalisé facilite les relations entre les personnes, mais il semble également nous conduire à quitter nos appartenances singulières. Le comble, c'est que la globalisation parvient à menacer tout à la fois la différence et l'universel. Cette tendance à l'uniformité est contraire en fait à la dignité humaine, car elle nie la richesse de chaque homme et de chaque pays dans sa diversité, son histoire, sa culture.
\end{abstract}

Christine Boutin

(De la mondialisation à l'universalisation: une ambition sociale)

Récemment, le Centre de Recherche en Langues et Cultures Européennes Comparées de L'Université de Lausanne nous a lancé un grand défi: il a posé la question de savoir si les termes "mondialisation" et "globalisation" sont équivalents. Cet article, inspiré par un regard multidisciplinaire, s'emploie à faire une révision conceptuelle des deux termes, une révision à partir du terme Weltliteratur qui a gagné du terrain sous la plume de Goethe. La discussion engendrée par l'auteur allemand anticipe le débat autours de problèmes soulevés par le processus de mondialisation/globalisation.

Au début du XIX siècle, Goethe avait déjà eu comme préoccupation constante d'articuler les notions de l'universel et du particulier sans établir une dichotomie mais en montrant la relation de complémentarité entre les deux termes. Comme nous le savons, Goethe avait l'esprit large relativement aux manifestations artistiques d'autres pays, il a souligné dans sa correspondance que le processus d'internationalisation était inévitable et a classifié la Weltliterature comme patrimoine commum de l'humanité.

Au premier regard, nous entendons le concept comme étant à peine synonyme d'une sorte de bibliothèque des chef-d'euvres mais si on examine l'origine du terme Weltliterature on s'apercoit d'emblée que le mot ne se borne pas au champ littéraire, c'est un concept qui apparaît dans plusieurs domaines de la production goethienne comme synonyme d'universalité. Le concept de Goethe s'élargit, c'est-à- dire, une littérature mondiale serait une littérature qui devrait se rapprocher d'un nouveau ethos universel. Le terme fonctionne aussi comme un link parmi les différentes littératures et les propres nations: 
World literature is, then, according to Goethe, the literature which serves as a link between national literatures and thus between the nations themselves, for the exchange of ideal values (...) It is a literary bridge over diving rivers, a spiritual highway over dividing mountains. It is an intellectual barter, a traffic in ideas between people, a literary market to which nations bring their intellectual treasures of Exchange. (STRICH,1949, p.14)

Le concept de Weltliterature émerge de l'observation de l'existence d'un constant processus d'effets réciproques parmi les littératures, il rend visible les transformations que les littératures nationales ont subi pendant les échanges universels. La théorie goethienne propose un dialogue avec l'autre, l'idée d'une littérature mondiale qui comprend également la diversité culturelle d'autres nations sans considérer d'autres cultures inférieures à la sienne ${ }^{1}$. En d'autres termes, le concept de Weltliterature cherche dans le national ce qu'il y a $\mathrm{d}^{\prime}$ universel et en sens inverse, cherche dans l'universel, ce qu'il y a de national. D'après, Luiza Lobo (1987,p.32) le terme Weltliterature peut être considéré comme un terme équivalent de Littérature Comparée.

Il est intéressant de remarquer que le concept de Goethe a mis l'accent sur le cosmopolitisme littéraire, sur la valorisation de la pluralité de littératures et de langues nationales, il a remarqué les gains théoriques des échanges culturels, la pensée de Goethe signale que nous ne pouvons pas analyser les identités culturelles et les littératures étrangères à partir $\mathrm{d}^{\prime}$ une notion de frontière fixe $\mathrm{c}^{\prime}$ est ce que fait justement la Littérature Comparée dont le concept de frontière est dépassable. Ce n'est pas par hasard que Todorov dans son ouvrage The morals of History (1991) a classifié Goethe comme le premier théoricien de l'interaction culturelle, selon le point de vue de Todorov le concept de Goethe a montré le chemin à l'idée de signification partagée.

Conscient de que son concept pourrait être entendu comme une forme de standardisation de différentes cultures, à partir de $1^{\prime}$ hégémonie de la culture plus riche comme dans le cas de la globalisation. En 1828, il a écrit un article pour la Revue Edinburgh Rewiens, où il a précisé son concept dans une perspective humaniste.En peu de mots, la vision goethienne du vaste monde, c'est la vision d'une Patrie amplifiée.

Aujourd hui, nous vivons dans un autre contexte bien différent de celui que Goethe a vécu. Certainement, on vit actuellement dans une société dite globale, ce qu'il faut considérer ce sont les impacts de ses idées globalisées sur les faits culturels, mais aussi sur les propres cultures, selon Helena Buescu:

Globalisation, village global sont des classifications que l'on rencontre un peu partout, des classifications disons à la mode, qui sont fréquemment utilisées pour décrire un monde apparemment uniformisé et homogénéisé, ou, peut-être mieux, uniformisable, surtout si l'on ne tient compte qu'à l'impact des médias et des nouvelles technologies, en particulier de celles qui nous font décrire notre société comme une société d'information.(BUESCU, 2002,p. 437)

L'expansion de la globalisation a réduit le monde, les connexions géographiques sont devenues aussi importantes que les connexions historiques, les nouvelles configurations politiques et économiques ont rédessiné le monde, ont approché les nations. Cette nouvelle configuration mondiale nous oblige à réarticuler les notions d'universel et de particulier proposés par Goethe.

L'apport de Goethe a été essentiel pour penser notre société contemporaine. Autrement dit, auteurs comme Alain Touraine, Christine Boutin, Helena Buescu, Jean Bessière, Renato Ortiz, Tania Franco Carvalhal, pour n’en citer que quelques uns, essaient 
tous de reprendre le débat et recontextualiser les termes globalisation, mondialisation et internationalisation.

Pour certains auteurs, comme Alain Touraine et Christine Boutin les termes globalisation et mondialisation sont employés comme synonymes. Suivant l'opinion de Touraine, la mondialisation (ou globalisation pour les anglo-saxons) est le processus d'ouverture de toutes les économies nationales sur un marché devenu planetáire. Spécialement dans son ouvrage intitulé Après la Crise (2010), l'auteur attire notre attention sur les effets, disons pervers, de la globalisation/mondialisation qui font disparaitre le social. En d'autres termes, il y a un silence surtout de la part des intellectuels par rapport à ce que Touraine appelle le "gangstérisme" financier, la dénationalisation de l'économie. Touraine s'accorde avec Goethe en ce sens que le processus d'universalisation préssupose la reconnaissance d'une culture plurale et encourage les échanges culturels.

En ce qui concerne la définition formulée par Christine Boutin, en son rapport intermédiaire de la République, l'ancien ministre entend la globalisation comme un synonyme d'américanisation et à l'exemple de Touraine, elle signale le mépris par le caractère social apporté par le processus de globalisation:

\begin{abstract}
Depuis les années 1970, le capitalisme financier s'est développé en conquérant la surface du globe, aidé en cela par les entreprises multinationales et les banques. Il a imposé au monde entier sa conception du libre-échange, fondée sur une vision occidentale du monde. Pour les pays riches, cette « occidentalisation » signifie souvent «américanisation», et se déroula la plupart du temps sans que les règles du commerce international ne prennent en compte la moindre considération à caractère social.(BOUTIN,2010,p. 14-15)
\end{abstract}

Dans son commentaire, nous remarquons déjá une critique explicite à l'impérialisme des États-Unis, n’oublions pas que l'impérialisme ne se limite pas aux armes, c'est aussi un ensemble d'idées, d'images et de répresentations. L'ancien ministre fait des reproches à ce monolinguisme ${ }^{2}$ imposé par les Etáts-Unis et qui a suscité plusieurs débats en France surtout en ce que touche à l'enseignement universitaire.

Je ne vais pas $\mathrm{m}^{\prime}$ allonger sur cette question, mais pour illustrer ce que vient de dire Boutin, c'est la polémique autour du projet de loi sur l'enseignement supérieur qui prévoit de faciliter la mise en place de cours en anglais et qui a provoqué plusieurs réactions ${ }^{3}$. Antoine Compagnon, professeur au Collège de France et à l'Université Columbia en interview au chat Le Monde.fr a exprimé son opinion, je dirais son insatisfation, par rapport à cette polique de la langue:

Pierrot: A Sciences Po Paris ou dans d'autres grandes écoles, il existe déjà des cursus totalement en anglais. Quelles leçons peut-on en tirer?

Compagnon: On peut en tirer la leçon que c'est inquiétant. Cela veut dire qu'on n'a pas besoin du français dans le monde global, qu'on considère qu'il est inutile dans les études puisqu'il ne servira à rien ensuite. C'est donc une atteinte à la dignité de cette langue.(COMPAGNON,2013,p.1)

La remarque de Compagnon est encore valable pour le contexte littéraire une fois que l'anglais ne peut pas s' instituer comme langue unique du globe parce qu'il n'existe pas un global man, la globalisation est un processus éminemment économique d'unification et de standardisation. Une littérature globalisée, ce n’est pas une littérature qui englobe d'autres littératures au contraire, le critère de jugement, d'exclusion serait économique pas esthétique. Une littérature globalisée exclurait l'idée de comparaison, puisque tous les écrivains, écrivant en anglais, rendraient compte d'un monde unique de realités et de rêves. 
La comparatiste portugaise Helena Buescu souligne les dangers de l'utilisation acritique, massive et apparemment "innocents" de la globalisation, un concept qui ne peut pas être considéré comme synonyme d' universel, le concept de globalisation essaie d'effacer la culture locale, $d^{\prime}$ homogénéiser les pratiques sociales:

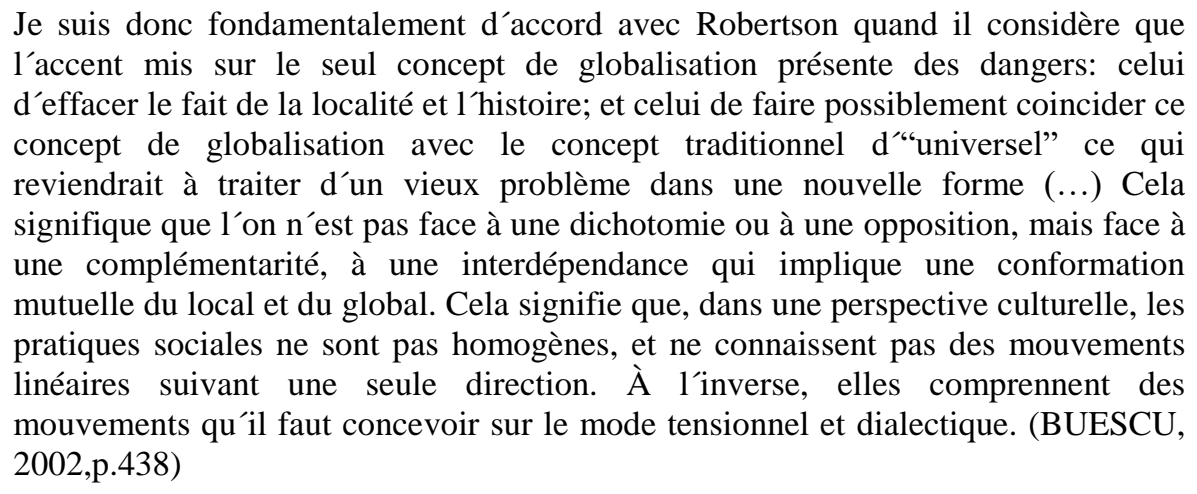

Le sociologue Renato Ortiz dans son ouvrage classique Mondialisation et culture (1994), distingue les termes global et mondial en employant le premier terme pour les processus économique et technologique, le second terme pour le domaine spécifique de la culture. Une culture mondialisée correspond à une civilisation dont la territorialité s'est globalisée, mais pas forcément les idées.

Tania Carvalhal, l'une des fondatrices de l'Association Brésilienne de Littérature Comparée, partage la même opinion de Renato Ortiz, elle soutient que les termes globalisation et mondialisation ne doivent pas être employés comme synonymes. D'après Carvalhal (2003), l'identité se construit comme un système complexe et élaboré de relations avec d'autres groupes, il y a toujours une relation de dépendance et d'interaction qui s'articule entre le particulier et l'universel.

Selon Jean Bessière, heureusement, il n'existe pas une homogénéisation des différences ni une assimilation des altérités, comme on pourrait supposer dans un processus de globalisation:

L'important n'est ni le constat ni la défense de l'identité et du multiculturalisme. Identité et multiculturalisme appartiennent à toute société, à toute culture, même si l'idéologie de telle société, de telle culture, les refuse (...)Le monoculturalisme est difficilement tenable et même concevable et, dans une culture, des particularismes culturels demandent toujours à être reconnus.(BESSIÈRE, 2002,p.445)

Ainsi, on peut dire que le processus de mondialisation culturel s'est complèté jusqu`a la fin du XX siècle par la globalisation économique, malgré la tentative d'uniformité, il y a eu lieu paradoxalement à l'augmentantion de l'affirmation de particularismes et on a vu que le monde ne peut pas être réduit à $l^{\prime}$ identique. L'émergence des particularismes est un symptôme du grand projet global qui est entré dans une profonde crise. On remarque cette crise surtout dans le domaine littéraire où les minorités se font entendre ${ }^{4}$.

Dans ce sens, le concept de Weltliteratur a démontré que les frontières politiques ne sont pas décisives pour imposer les frontières culturelles. Si c'était comme ça, la Littérature des États-Unis -pays qu' on peut dire berceau de la globalisation, l'un des pays plus riches, jouirait d'une grande renommées.

En contrepartie, en Irlande on vérifie un autre phénomène, un pays disons pauvre par rapport aux États-Unis, mais célèbre par ses écrivains. La littérautre irlandaise est reconnue dans le monde. L'Irlande s'enorgueillit de n'avoir pas moins de quatre prix Nobel de 
littérature: William Butler Yeats, George Bernard Shaw, Samuel Beckett et Seamus Heaney, ce qui est véritablement étonnant pour une petite île. La Littérature Irlandaise est un bon exemple pour démontrer que les hégémonies politiques et économiques, traits caractéristiques de la globalisation, ont du mal à s'imposer dans l'espace littéraire. Les Étaits-Unis ont su exporté leur modèle écononomique, technologique mais ont du mal à exporter leur capital littéraire.

En profitant des exemples donnés, dire que le Prix Nobel de Littérature s'est globalisé, est à mon avis une affirmation troublante.Le Prix Nobel de Littérature dès son origine ne vise pas proprement l'économique, donc il ne représente pas la littérature globalisée, si on entend par globalisée une littérature de massification, de standardisation, le Nobel vise une littérature dont la vocation est universelle, ce qui est tout à fait différent.

Donc, parler d'une littérature globalisée ne me semble pas approprié, l'espace littéraire a ses propres lois, les lois du texte. La littérature a une capacité de mettre en relation des espaces hétérogènes, d'opérer de nouvelles spatialisations, l'espace littéraire se constitue comme un espace polyphonique où toutes les voix se matérialisent dans la page écrite ${ }^{6}$.

Les quinze dernières années, on a assisté dans le contexte comparatiste, à la réactivation autour de la notion de Weltliteratur qui a eté reprise par l'anglicisation du terme World Literature (Littérature Monde) qui suggère l'américanisation des études littéraires en général. Les débats autour de la Littérature Monde (World Literature) nous permettent du moins approfondir la conscience du caractère transversal des phénòmenes littéraires et démontrer qu'il y a une relation de complémentarité, comme disait Goethe, parmi les différentes littératures.

La réflexion développée par Goethe devient centrale car elle nous aide à comprendre les transformations que les littératures ont subi à partir des modèles importés.L' Histoire Littéraire de chaque pays, devient exemplaire pour vérifier que les impositions culturelles ont engendré une expression littéraire différente. Le parcours de chaque littérature est fait de rencontres et d'incorporations et aussi à l'inverse, de rencontres manquées et de refus.

Le concept de Weltliteratur est sans doute l'un des concepts fondateurs de la discipline de Littérature Comparée. La Littérature Comparée en sa constante auto-réflexion méthodologique et en son analyse sur la themátique de la globalisation, souligne que la Littérature Monde (World Literature) ne pourra jamais perdre de vue son horizon de recherche c'est-à-dire, la notion de littérature comme une archive de différences.Dès son institutionalisation comme discipline, la Literature Comparée a comme principe fondamental le respect à la culture des autres nations.

Si l'altermondialisation ${ }^{7}$, soutenue par quelques comparatistes, n'est pas la meilleure alternative pour contrôler les effets de la globalisation, c'est déjà un mouvement vers l'humanisme, vers une mondialisation plus démocratique, nous indique que peut-être un Welthumanisme peut surgir de cette discussion.

On a essayé de réoxygéner le concept de Goethe, un concept qui permet le développément d'une pensée du passé, indissociable d'une pensée de l'avenir, nous avons essayé de montrer dans ce travail que la Littérature Comparée, dans l'ensemble des théories qu'elle soutient est une voix qui se soulève contre les risques qui peuvent accompagner le processus de la globalisation surtout sur le plan culturel, en soutenant un humanisme et un cosmopolitisme littéraires, soulignant l'autonomie de l'espace littéraire par rapport aux hégénomies politiques et économiques imposées par la globalisation.

En somme, le grande défi qui s'impose aux comparatistes contemporains c'est d'évaluer la dimension de la rupture épistémologique provoquée par la dissemination de la globalisation et penser ,dans ce contexte, aux nouvelles expériences épistémologiques qui se présentent dans le monde issues du processus, spécialement à travers le texte littéraire parce 
que le texte littéraire est la mise en forme esthétique des conflits culturels, c'est un espace privilégié pour évidencier les conflits.Sûrement, d'autres mondes sont possibles et la littérature avec sa conscience toujours émancipatrice, essayera de nous les faire connaitre.

\section{Comparative Literature: An Insurgent Voice in the Context of Globalization}

ABSTRACT: The Comparative Literature confronts different literatures and subjects, by putting in local and national aspects in perspective, it has shown us that literature, though in a context of globalization, remained a sphere of resistance; that is, the literary text is not wholly subservient to models imposed by globalization, as the literary text always has a critical and deforming view with regard to such models.

Key-words: comparative literature; world literature; Welt-humanism; globalization.

\section{NOTES}

${ }^{1}$ Cet éloge à la différence, ce discours en faveur de la tolérance, ce sont des idées présentes dans les Essais de Michel de Montaigne. THIBAUDET, Albert.(ORG).Michel de Montaigne. Essais. Paris: Gallimard, 1950.p.243.

${ }^{2}$ «C'est en faisant fond sur ce fond qu'opère le monolinguisme imposé par l'autre, ici par une souveraineté d'essence toujours coloniale et qui tend, représsiblement et irrepréssiblement, à réduire les langues à l'Un, c'està-dire à l'hégémonie de l'homogène. On le vérifie partout où dans la culture cette homo-hégémonie reste à l'oeuvre, effaçant le pli et mettant le texte à plat». DERRIDA, Jacques Le monolinguisme de l'autre ou la prothèse d'origine. Paris: Éditions Galilée, 1996.p.69.

${ }^{3}$ L'historien Cristophe Charle dans son Histoire des Universités, parle plus profondement de cette domination anglophone dans l'espace de l'enseignement universitáire. Sans doute, la question est troublante, il faut faire face à ce type de massification, le français et d'autres langues étrangères courrent le risque d'être chaque fois plus marginalisées. CHARLE, Christophe.Histoire des Universités. Paris: PUF,2013.

${ }^{4}$ La littérature est toujours une réflexion sur la culture, dans une perspective transculturelle les éléments culturels sont retravaillés. Toute création artistique fonctionne selon ce principe de la transformation constante des modèles disponibles à l'écrivain. Il y a toujours un lien très étroit entre l'identité et le contexte. En d'autre mots, il y a toujours une confrontration des altérités c'est-à-dire, le texte littéraire n'est pas tout fait soumis aux modèles, le littérature a toujours un regard critique et déformant par rapport à ces modèles. Selon Maurice Blanchot:«L'essence de la littérature, c'est d'échapper à toute détermination essentielle, à toute affirmation qui la stabilise ou même la réalise: elle n'est jamais déjà là, elle est toujours à retrouver ou à réinventer”.'BLANCHOT, Maurice. Le livre à venir. Paris: Gallimard, 1959.p.305.

${ }^{5}$ Il est intéressant de vérifier que la Lost Generation ( Génération Perdue) dont les écrivains plus représentatifs sont Ernest Hemingway, Gertrude Stein, Francis Scott Fitzgerald, John Steinbeck et T.S.Eliot, c'est la génération américaine qui a justement vécu dans l'exil et qui a connu un énorme succès auprès du public. Lire ROYOT, Daniel. La littérature américaine, P.U.F. 'Que sais-je ?', 2004, p. 123.

${ }^{6}$-Malgré les critiques par rapport à 1 ouvrage de Pascale Casanova, République Mondiale des lettres, je suis d'accord avec 1'idée préliminaire de cet ouvrage, où Pascale affirme que la "République Mondiale des Lettres est un univers dans lequel s'affronteraient territoires et espaces littéraires indépendamment des tracés politiques, l'espace littéraire est un espace de configuration complexe”. CASANOVA, Pascale. République Mondiale des lettres. Paris:Seuil, 2008.p.20.

${ }^{7} \mathrm{Ce}$ concept a été forgé au cours de l'année 2001. Altermondialisation est le concept qui rend compte de la démarche de construction d'alternatives au paradigme dominant — depuis deux décennies environ — de la globalisation économique, financière et informationnelle. Avec son émergence, il ne s'agit plus d'une simple et systématique contestation de cette globalisation (ce que l'on désigne par antimondialisation), mais bien de sa mise en perspective critique, de son éloignement radical, ainsi que de l'expérimentation de nouvelles propositions, de nouveaux modèles d'organisation économique, sociale, politique et culturelle. Voir à ce propos Revues Mouvements et Transversales (coord.)Où va le mouvement altermondialisation? Paris: LaDécouverte, 2003.

\section{BIBLIOGRAPHIE}

IPOTESI, JUIZ DE FORA, v.22, n.1, p. 70-77, jan./jun. 2018 
BESSIÈRE, Jean \& ANDRÉ, Sylvie. «Notes pour une typologie des littératures occidentales suivant le jeu de l'identité et de la différence avec un coda sur Édouard Glissant». In: Multiculturalisme et identité en littérature et en art. Paris: L'Harmattan,2002.

«Théorie et critique littéraires contemporaines,cultures nationales et thèses transnationales.Paradoxes de la pensée de l'universel et du culturel». In: COUTINHO, Eduardo F. (ORG.).Fronteiras imaginadas: cultura nacional / teoria internacional, Rio de Janeiro, Aeroplano, 2001.p.13-47.

BLANCHOT, Maurice. Le livre à venir. Paris: Gallimard, 1959.

BOUTIN, Christine. De la mondialisation à l'universalisation: une ambition sociale. Rapport intermédiaire au Président de la République .France: Collections des rapports officiels, 2010.p.17.

BUESCU, Helena Carvalhão. Littérature Comparée et Globalisation In: Multiculturalisme et identité en littérature et en art. Paris: L'Harmattan,2002.

.Experiência incomum e boa vizinhança. Literatura Comparada e Literatura-Mundo.Porto: Porto Editora, 2013.

CARVALHAL, Tania Franco. O próprio e o alheio: ensaios de Literatura Comparada. São Leopoldo: Unisinos, 2003.

CASANOVA, Pascale. République Mondiale des lettres. Paris:Seuil, 2008.

CHARLE, Christophe.Histoire des Universités. Paris: PUF,2013.

COMPAGNON, Antoine, Enseignement de l'anglais à l'Université: "Il faut des mesures ciblées, pas un chèque en blanc" Disponível em: «http://www.lemonde.fr». Acesso em:16/maio. 2013.

DERRIDA, Jacques Le monolinguisme de l'autre ou la prothèse d'origine. Paris: Éditions Galilée, 1996.

ECKERMANN, Johann Peter. Conversações de Goethe com Eckermann.Trad. Luís Silveira. Porto: Livraria Tavares Miranda, 1947.p.161.

HEISE, Eloá. Goethe, um teórico da transnacionalidade.In: Revista Brasileira de Literatura Comparada, $\mathrm{n}^{\circ}$ 5, Salvador ,2000.p.77-84.

LACLAU, Ernesto. Emancipación y diferencia. Buenos Aires:Esparsa-Calpe,1996.

LOBO, Luíza. Teorias poéticas do Romantismo. Rio de Janeiro: Editora da UFRJ, 1987 (Série Novas Perspectivas, 20).

ORTIZ, Renato. Mundialização e cultura. São Paulo: Brasiliense, 1994.

REVUES MOUVEMENTS ET TRANSVERSALES (coord.). Où va le mouvement altermondialisation? Paris: La Découverte, 2003.

ROYOT, Daniel. La littérature américaine. Paris: P.U.F. 'Que sais-je ?', 2004.

STRICH, Fritz. Goethe and World Literature. London: Routledge \&Kegan Paul, 1949. 
THIBAUDET, Albert.(ORG).Michel de Montaigne. Essais. Paris: Gallimard, 1950.

TODOROV, T. The morais of History. Minneapolis: University of Minnesota Press, 1991. TOURAINE, Alain. Après la crise. Paris: Seuil, 2010.

Enviado em 26 de junho de 2018.

IPOTESI, JUIZ DE FORA, v.22, n.1, p. 70-77, jan./jun. 2018 